\title{
The Effect of Water Models on Desalination Through Graphene Nanopores
}

\author{
Vishnu Prasad K ${ }^{1}$, Sridhar Kumar Kannam ${ }^{2}$, Remco Hartkamp ${ }^{3}$, Sarith P Sathian ${ }^{1}$ \\ ${ }^{1}$ Department of Applied Mechanics, Indian Institute of Technology Madras, \\ Chennai-600036, India. \\ vishnu.prasad.1729@gmail.com; sarith@iitm.ac.in \\ ${ }^{2}$ Swinburne University of Technology, \\ Hawthorn, Victoria-3122, Australia \\ urssrisri@gmail.com \\ ${ }^{3}$ Process and Energy Department, Delft University of Technology, \\ Leeghwaterstraat 39, 2628 CB Delft, Netherlands \\ r.m.hartkamp@tudelft.nl
}

\begin{abstract}
Nanoporous carbon materials are extensively studied for various separation applications. Among them, water desalination by means of Reverse Osmosis (RO) stands out due to it's large socio-economic relevance. Many studies are carried out in this area both computationally and experimentally. In computational studies the water simulated using different water models are prone to produce inconsistent results. In this study water desalination through hydrogen functionalized graphene nanopore is studied using different water models (SPC, SPC/E, TIP3P, TIP4P/2005). Up to 81\% difference was observed in the flux estimates among the models. The water permeation rate was found to be closely related to the bulk transport properties of the simulated water.
\end{abstract}

Keywords: Nanofluidics, Desalination, Molecular Dynamics, Reverse Osmosis, Graphene, Nanopore.

\section{Introduction}

Availability of fresh water is a growing global concern. With the present rates of socio-economic developments across the globe, the natural fresh water resources will soon be deficient in meeting the requirement. It is estimated that by 2026 two third of the global population will be water strained [1]. Against this background, water desalination promises to be a solution to the problem [2] as the oceans hold $95 \%$ of the earth's water. Using seawater desalination, water that is external to the planet's natural water cycle could be made available for human use.

Desalination using membrane based separation techniques are much efficient compared to thermal based techniques, particularly Reverse Osmosis (RO), a technique that constitutes nearly half the installed desalination capacity across the globe [3]. In RO, the seawater is passed through a semi-permeable membrane at high pressures such that the ions get filtered out. The performance of RO primarily depends on (a) the rate of water permeation, and (b) the ion rejection of the membrane. Investigations are carried out on novel membrane materials with the objective to improve the water permeation rate and ion rejection [4].

Nanoporous carbon materials show unprecedented water permeation through them owing to their hydrophobic nature [5]. These materials are hence a strong candidates for replacing the existing RO membranes. Studies are held on carbon nanotubes (CNTs) [6], graphene oxide (GO) [7] and graphene nanopores [8] for water desalination applications. For example, Suk et al. [9] studied the water flow through graphene nanopores and formulated a corrected Hagen-Poiseuille equation based on continuum description of the flow. In a pioneering study, Cohen-Tanugi and Grossman found a large water permeation across nanoporous graphene membranes $[10,11]$. They found that water permeation through the graphene nanopores were several orders higher than that achieved by commercially available RO membranes [10]. Konatham et al. [12] studied the energy landscape of water and ions across graphene nanopores and found that the water permeation is affected by the presence of functional groups and the pore size. O'hern et al. [13] experimentally studied water desalination across nanoporous graphene. They were successful in filtering $\mathrm{MgSO}_{4}$, while the $\mathrm{NaCl}$ passed through due to tears in the sheet. 
Surwade et al. [14] successfully filtered out $\mathrm{NaCl}$ from water using nanoporous graphene membranes created using chemical vapour deposition (CVD) followed by exposure to oxygen plasma. Chen and Yang [15] conducted studies on pyridinic nitrogen doped graphene nanoporous membranes and found a high water flux for hydroxyl functionalized nanopores in agreement with previous studies $[10,12]$.

There exist quantitative inconsistencies among the results from above studies arising mainly from the difference in simulation methods used in the studies. For instance, the simulation of water using different water models were found to have an effect. Liu and Patey $[16,17,18]$ found considerable difference in the flux across CNTs (diameters $12.35 \AA$, $10.98 \AA$, and $8.23 \AA$ ) using the water models TIP3P, TIP4P/2005, and SPC/E. They attributed the flux difference in $\mathrm{CNT}(6,6)($ diameter $8.23 \AA$ ) to the activation energy of entry into the CNTs and the difference in the bulk properties of the water simulated using the models. This indicates that water desalination across graphene nanopores could also be influenced by the water model used. Further, the effect of water models and the physical properties of simulated water on desalination needs a deeper understanding.

Here, we investigate the effect of water model used on desalination across hydrogen functionalized graphene nanopores. We use the SPC, SPC/E, TIP3P, and TIP4P/2005 water models for the study. We quantify the water permeation across the membrane and compare it with the bulk transport properties of the water obtained using different models. Our results indicate that water permeation through graphene nanopores is largely dependent on the bulk transport properties of the simulated water.

\section{Methodology}
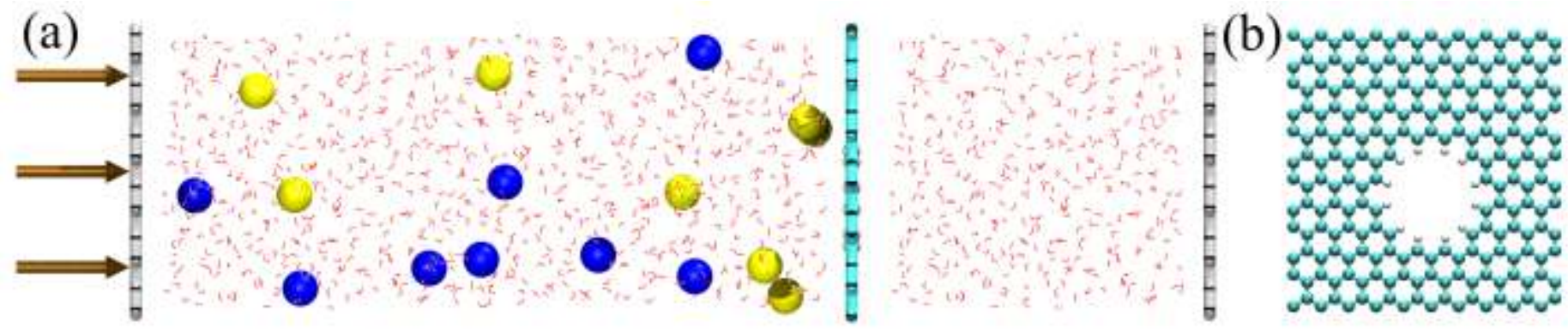

Fig. 1: (a) The system used for the study. The cyan sheet represents the pore sheet. The silver sheets at the ends represent the pistons.

Water molecules are represented as tiny red angles for clarity. The yellow spheres represent the sodium ion and the blue spheres represent the chloride ion. The arrows indicate application of pressure. (b) Hydrogen functionalized nanopore used for the study. The cyan spheres represent the carbon atoms and the white spheres represent the hydrogen atoms.

Hydrogen functionalized graphene nanopore of area $23 \AA^{2}$ was simulated [10] (see Figure 1). The feed reservoir (60 A long) was filled with 877 water molecules, and 8 sodium and 8 chloride ions (salinity $\approx 0.5 \mathrm{M}$ ). The permeate reservoir (30 A long) was filled with 395 water molecules. Plain graphene sheets were placed at the ends of the reservoirs as pistons for pressurization. The force field parameters for the graphene sheets, ions and the functional groups were taken from Cohen-Tanugi and Grossman [10]. The parameters for water were the standard parameter values of the water models investigated. The SHAKE algorithm was used to keep the bonds and angles of the water molecules rigid. Periodic boundary conditions were applied in all directions. The simulations were carried out using the LAMMPS package [19].

The energy of the pore functionals were first minimized and and then the energy of the entire system was minimized. Equilibration of the system was carried out for $1 \mathrm{~ns}$ in the NVT ensemble at $300 \mathrm{~K}$ with the application of 0.101325 $\mathrm{MPa}(1 \mathrm{~atm})$ pressure in the feed and permeate reservoirs using pistons. During the production run, that lasted for $5 \mathrm{~ns}$, the feed side pressure was increased to $200 \mathrm{MPa}$ causing water permeation across the nanopore. Three independent simulations with different initial conditions were carried out for each case and the mean values were taken. 


\section{Results and Discussions}

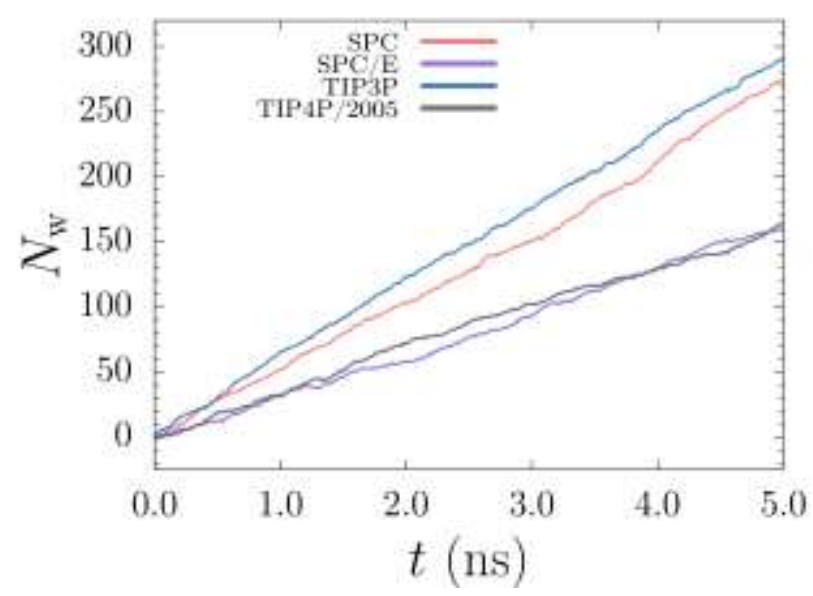

Fig. 2: Number of water molecules crossing the membrane with time. The results are average of three independent simulations.

Figure 2 shows the number of water molecules $\left(N_{\mathrm{w}}\right)$ crossing the membrane with time $(t)$. The slope of the curve represents the permeation rate. There is up to $81 \%$ difference between the permeation rates shown by different models. SPC and TIP3P models shows a higher permeation rate compared to SPC/E and TIP4P/2005. The results are in-line with previous studies $[16,17]$. For all the models, there was zero ion permeation, in agreement with previous studies [10]. Further, the permeation rates were found to be directly related to the partial charge distribution of the water models [20]. For example, SPC and TIP3P have a smaller partial charge values for oxygen and hydrogen atoms and produced a higher water flux compared to SPC/E and TIP4P/2005.

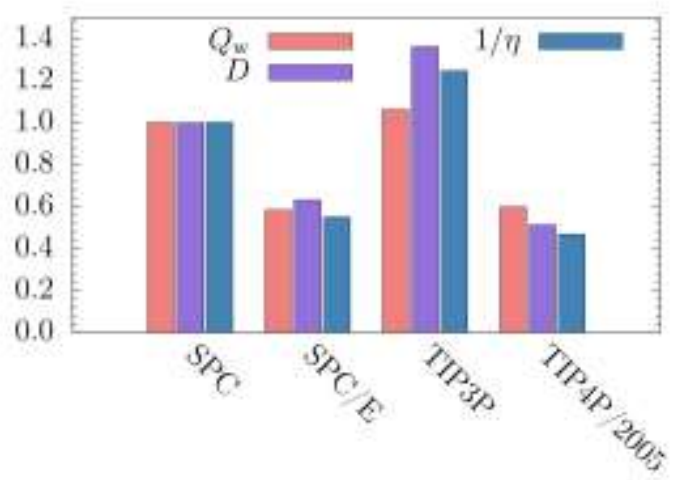

Fig. 3: Comparison of the permeation rate with the bulk transport properties of water exhibited by different water models. The values are normalized with respect to SPC model values.

Figure 3 shows the comparison between the permeation rate $\left(Q_{\mathrm{w}}\right)$, the diffusion coefficient $(D)$ and the inverse of shear viscosity $(1 / \eta)$ shown by the water models, all normalized with respect to SPC model values. Table 1 provides the absolute values of the results shown in figure 3. There exists a close correlation between the permeation rate and the bulk transport properties of the simulated water. The diffusion coefficient and the permeation rate are directly correlated. Also, the shear viscosity of the simulated water is inversely correlated with the permeation rate. This is in conformance with the StokesEinstein relationship for fluid continuum. The results are also in agreement with previous studies [17]. 
Table 1: The permeation rate $\left(Q_{\mathrm{w}}, \mathrm{ns}^{-1}\right)$, diffusion coefficient $\left(D, 10^{-5} \mathrm{~cm}^{2} \mathrm{~s}^{-1}\right)$, and shear viscosity $(\eta$, mPas) shown by the water models. The shear viscosity and diffusion coefficient for SPC were taken from Wu et al. [21] (1.013 bar, $298.15 \mathrm{~K}$ ). For the other models, the values were taken from Vega and Abascal [22] (1 bar, $298 \mathrm{~K})$.

\begin{tabular}{|l|c|c|c|}
\hline Water Model & $\boldsymbol{Q}_{\mathbf{w}}$ & $\boldsymbol{D}$ & $\boldsymbol{\eta}$ \\
\hline SPC & 55.0 & 4.02 & 0.40 \\
\hline SPC/E & 32.06 & 2.54 & 0.72 \\
\hline TIP3P & 58.33 & 5.48 & 0.32 \\
\hline TIP4P/2005 & 32.86 & 2.06 & 0.85 \\
\hline Experimental Value & NA & 2.30 & 0.89 \\
\hline
\end{tabular}

In order to rule out the possibility that the flux difference observed arose from the difference in pore water interaction of different models, we quantified the residence time of water molecules near the pore functional groups. The residence time of the water molecules $\left(t_{\text {res }}\right)$ that were within $3.5 \AA$ (defined as near) from the functional atoms was calculated. Figure 4 compares the average residence time of water molecules near the pore functionals with the permeation rate. The values are normalized with respect to SPC values and the inverse of residence time is plotted to illustrate the correlation better. The residence time shown by all the models are similar indicating similar interaction between the water and the functional atoms across all the models. Further, flux obtained by simulating the nanopores without charges on the functional groups also show good correlation with the bulk transport properties. This confirms that the bulk properties of the simulated water are the major contributing factor to the difference in permeation rate [17].

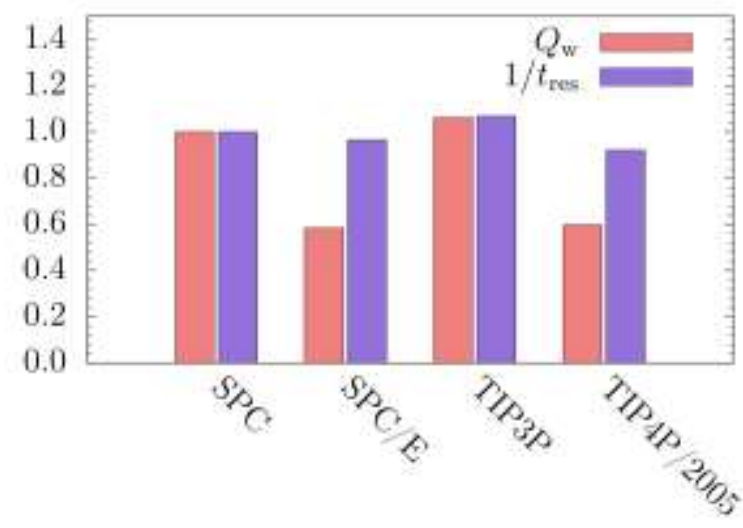

Fig. 4: Comparison of the permeation rate with the average residence time of water molecules near the nanopore functional atoms. All the values are normalized with respect to SPC values. The inverse of residence time is plotted for illustrate the correlation better.

\section{Conclusion}

Water desalination through hydrogen functionalized graphene nanopores was studied using different water models. The water permeation was found to be influenced by the bulk transport properties of the simulated water. The residence time of water near the pore functionals were similar for all the models suggesting that the water permeation is mainly influenced by the bulk transport properties of the simulated water. The results indicate that a higher diffusion coefficient and/or lower shear viscosity of the solvent would result in an enhanced water flux. For RO desalination systems, this may be achieved by changing the temperature [23] or by disrupting the hydrogen bonds of water [24, 25].

\section{Acknowledgements}

We thank the financial support received from Department of Science and Technology (DST), Government of India, under Water Technology Initiative (Project Number: DST/TM/WTI/2K15/84(G)). We thank David Cohen- Tanugi, EMBR labs, Cambridge, USA, for sharing with us the nanopore geometry and other details for this study. 


\section{References}

[1] R. F. Service, "Desalination freshens up," Science, vol. 313, no. 5790, pp. 1088-1090, 2006.

[2] M. Elimelech and W. A. Phillip, "The future of seawater desalination: Energy, technology, and the environment," Science, vol. 333, no. 6043, pp. 712-717, 2011.

[3] I.G. Wenten and Khoiruddin, "Reverse osmosis applications: Prospect and challenges," Desalination, vol. 391, pp. 112-125, 2016.

[4] Luda Wang, Michael S. H. Boutilier, Piran R. Kidambi, Doojoon Jang, Nicolas G. Hadjiconstantinou, and Rohit Karnik, "Fundamental transport mechanisms, fabrication and potential applications of nanoporous atomically thin membranes," Nat. Nanotechnol., vol. 12, no. 6, pp. 509-522, 2017.

[5] Hemant Kumar, Biswaroop Mukherjee, Shiang-Tai Lin, Chandan Dasgupta, A. K. Sood, and Prabal K. Maiti, "Thermodynamics of water entry in hydrophobic channels of carbon nanotubes," J. Chem. Phys., vol. 134, no. 12, pp. 124105, 2011.

[6] Gerhard Hummer, Jayendran C Rasaiah, and Jerzy P Noworyta, "Water conduction through the hydrophobic channel of a carbon nanotube," Nature, vol. 414, no. 6860, pp. 188-190, 2001.

[7] R. R. Nair, H. A. Wu, P. N. Jayaram, I. V. Grigorieva, and A. K. Geim, "Unimpeded permeation of water through helium-leak-tight graphene-based membranes," Science, vol. 335, no. 6067, pp. 442-444, 2012.

[8] Khaled A. Mahmoud, Bilal Mansoor, Ali Mansour, and Marwan Khraisheh, "Functional graphene nanosheets: The next generation membranes for water desalination," Desalination, vol. 356, pp. 208-225, 2015.

[9] M. E. Suk and N. R. Aluru, "Molecular and continuum hydrodynamics in graphene nanopores," RSC Adv., vol. 3, no. 24, pp. 9365, 2013.

[10] David Cohen-Tanugi and Jeffrey C. Grossman, "Water desalination across nanoporous graphene," Nano Lett., vol. 12, no. 7,pp. 3602-3608, 2012.

[11] David Cohen-Tanugi and Jeffrey C. Grossman, "Water permeability of nanoporous graphene at realistic pressures for reverse osmosis desalination," J. Chem. Phys., vol. 141, no. 7, pp. 074704, 2014.

[12] Deepthi Konatham, Jing Yu, Tuan A. Ho, and Alberto Striolo, "Simulation insights for graphene-based water desalination membranes," Langmuir, vol. 29, no. 38, pp.11884-11897, 2013.

[13] Sean C. O’Hern, Michael S. H. Boutilier, Juan-Carlos Idrobo, Yi Song, Jing Kong, Tahar Laoui, Muataz Atieh, and Rohit Karnik, "Selective ionic transport through tunable subnanometer pores in single-layer graphene membranes," Nano Lett., vol. 14, no. 3, pp. 1234-1241, 2014.

[14] Sumedh P. Surwade, Sergei N. Smirnov, Ivan V. Vlassiouk, Raymond R. Unocic, Gabriel M. Veith, Sheng Dai, and Shannon M. Mahurin, "Water desalination using nanoporous single-layer graphene," Nat. Nanotechnol., vol. 10, no. 5, pp. 459-464, 2015.

[15] Qi Chen and Xiaoning Yang, "Pyridinic nitrogen doped nanoporous graphene as desalination membrane: Molecular simulation study," J. Membr. Sci., vol. 496, pp. 108-117, 2015.

[16] L. Liu and G. N. Patey, "Simulations of water transport through carbon nanotubes: How different water models influence the conduction rate," J. Chem. Phys., vol. 141, no. 18, pp. 18C518, 2014.

[17] L. Liu and G. N. Patey, "Simulated conduction rates of water through a $(6,6)$ carbon nanotube strongly depend on bulk properties of the model employed," J.Chem. Phys., vol. 144, no. 18, pp. 184502, 2016.

[18] L. Liu and G. N. Patey, "A molecular dynamics investigation of the influence of water structure on ion conduction through a carbon nanotube," J. Chem. Phys., vol. 146, no. 7, pp. 074502, 2017.

[19] Steve Plimpton, "Fast parallel algorithms for short-range molecular dynamics," J. Comput. Phys., vol. 117, no. 1, pp. $1-19,1995$.

[20] Vishnu Prasad K., Sridhar Kumar Kannam, Remco Hartkamp, and Sarith P. Sathian, "Water desalination using graphene nanopores: influence of the water models used in simulations," Phys. Chem. Chem. Phys., vol. 20, no. 23, pp. 16005-16011, 2018.

[21] Yujie Wu, Harald L. Tepper, and Gregory A. Voth, "Flexible simple point-charge water model with improved liquidstate properties," J. Chem. Phys., vol. 124, no. 2, pp. 024503, 2006. 
[22] Carlos Vega and Jose L. F. Abascal, "Simulating water with rigid non-polarizable models: a general perspective," Phys. Chem. Chem. Phys., vol. 13, no. 44, pp. 19663, 2011.

[23] Kazimierz Krynicki, Christopher D Green, and David W Sawyer, "Pressure and temperature dependence of selfdiffusion in water," Faraday Discuss. Chem. Soc., vol. 66, pp. 199-208, 1978.

[24] Hitoshi Ohtaki, "Effects of temperature and pressure on hydrogen bonds in water and in formamide," J. Mol. Liq., vol. 103, pp. 3-13, 2003.

[25] Zhaohui Wang, Yoonsoo Pang, and Dana D. Dlott, "Hydrogen-bond disruption by vibrational excitations in water," J. Phys. Chem. A, vol. 111, no. 17, pp. 3196-3208, 2007. 